\title{
A Navigation System for Computer Assisted Unicompartmental Arthroplasty
}

\author{
Maurilio Marcacci ${ }^{2}$, Oliver Tonet ${ }^{1}$, Giuseppe Megali ${ }^{1}$, Paolo Dario ${ }^{1}$, \\ Maria Chiara Carrozza ${ }^{1}$, Laura Nofrini ${ }^{2}$, Pier Francesco La Palombara ${ }^{2}$ \\ ${ }^{1}$ MiTech Lab, Scuola Superiore Sant'Anna, Pisa, Italy \\ peppe@mail-arts.sssup.it \\ oly@sssup.it \\ dario@arts.sssup.it \\ chiara@arts.sssup.it \\ http://www-mitech.sssup.it/ \\ Biomechanics Lab, Istituti Ortopedici Rizzoli, Bologna, Italy \\ \{M.Marcacci, L.Nofrini, F.La.Palombara\}@biomec.ior.it \\ http://www.ior.it/biomec/
}

\begin{abstract}
This work presents an overview of a prototype navigation system for computer assisted unicompartmental arthroplasty. The navigation system allows the surgeon to use a minimally invasive surgical technique, solving the problems due to a restricted knee exposure with an augmented reality environment. The key feature of the system is an interactive graphical interface in which a 3D model of the patient's joint and two 2D projections (frontal and lateral) are shown together with the model of a sensorized custom surgical cutting guide. The position of the sensorized instruments are tracked in realtime by an optoelectronic localizer and reproduced in the virtual scene. The 3D model of the joint is reconstructed from a preoperative CT/MRI data set and matched to the actual anatomy by means of an ICP-based non-fiducial registration algorithm. The prototype system has been positively evaluated by a selected group of skilled orthopaedic surgeons.
\end{abstract}

\section{Introduction}

Unicompartmental knee arthroplasty (UKA) is a kind of intervention that offers several advantages for the treatment of single compartment joint disease. Even if in the past there has been a lack of general agreement on how successful UKA has been, recently, clinical studies have reported satisfactory results [1][2] mainly depending on a correct patient selection criteria, and they have shown that in some cases UKA can also be considered a valid alternative procedure to high tibial osteotomy [3][4].

Traditional surgical techniques for UKA require numerous and difficult to use instruments, a large exposure area and patellar dislocation to determine the correct placement of the components, and its invasiveness is similar to total knee arthroplasty one. 
Recently, minimally invasive surgical techniques for UKA (RepicciII, , MillerGalante system) have been proposed: these procedures involves smaller incision, minimal blood loss, preservation of normal tissue, reduced pain, and shortens recovery and rehabilitation times[5]. However, the reduced exposure area leads to a hard retrieval of the anatomical landmarks used to correctly position the prosthesis components, requiring considerable surgical dexterity and skill. In particular the Repicci II technique has been criticize because it relies on freehand surgery.

Augmented reality systems can be useful to overcome the limitation due to the restricted exposure enhancing the surgical skill.

We propose a new computer-assisted approach to help the surgeon to solve problems related to minimally invasive UKA. Our methodology is based on the use of an intraoperative navigation system and of a reduced set of surgical instruments. In the following paragraphs we will describe the system showing the main advantages it offers in comparison with the other existing techniques.

\section{Material}

\subsection{Equipment}

The described procedure needs the standard surgical equipment for monitoring the patient, a PC for running the navigation system (the central control unit we use is an Intergraph TDZ 2000 GX1 workstation (pentium II xeon $450 \mathrm{MHz}, 128$ MB RAM, Windows NT 4.0) with RealiZm II 3D graphics board), a commercial optical localizer (FlashPoint 5000 system, Image Guided Technologies Inc.), three sensorized sterilizable frame with four IR LEDs each and a custom cutting guide.

The optical localizer. The optical localizer is based on three cameras located on a stand placed over the surgical scene, which defines the coordinate reference frame of the operating room. The cameras detect the IR pulses emitted by LEDs placed on rigid frames fixed to the objects to track. The control unit of the localization system computes the coordinates of the LED positions by means of geometrical triangulation and determines the position and orientation of the objects present in the scene, with an accuracy better than $0.5 \mathrm{~mm}$. This localizer is provided with a probe digitizer that can be used to acquire points in the working area.

In the surgical room, the optical localizer is positioned over the head of the patient so that it does not encumber the surgeon in the operating area.

The sensorized frames. Three sterilizable frames are used to track the objects in the operating room to reproduce their movements in the virtual scenary.

One frame is fastened to the femur in the distal frontal third of the bone. Another frame is fastened to the tibia in the proximal lateral third of the bone. The last frame is fixed on a custom cutting guide, designed on purpose for this surgical system (fig.1). 
The cutting guide. The cutting guide is a tool consisting of a small metal block and of a bar connecting the block with the sensorised frame. The block is $4 \times 2 \times 1 \mathrm{~cm}$ and it is provided with two holes $3.2 \mathrm{~mm} \varnothing$ to secure it to the bone with two pins.

The design of the new surgical tool has been outlined in collaboration with expert surgeons in order to satisfy the new surgical requirements of minimal invasiveness (fig. 1).

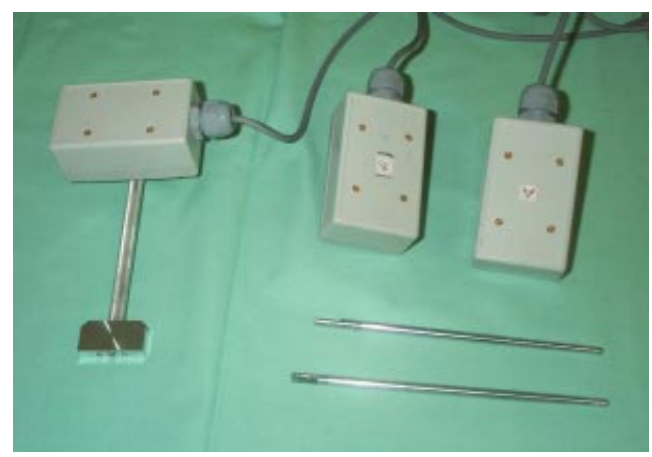

Fig. 1. The sensorized frames

\section{2 .2 The Navigation System Software}

The navigation system has been developed by MiTech Lab of Scuola Superiore Sant'Anna (Pisa, Italy), in collaboration with Biomechanics Lab of Istituti Ortopedici Rizzoli (Bologna, Italy)[6].

The navigation system includes a registration module for matching preoperative data, i.e. the virtual model of the anatomical structures, and intraoperative data, i.e. the actual patient's anatomy, a tracking module for real time monitoring of the position of the surgical tools and anatomical structures present in the operating room, and a graphical human-machine interface that displays the enhanced reality scenario based on a 3D model of patient's limb computed from CT/MRI. The navigation system has been developed in C++ language for Windows NT 4.0 platform, using Microsoft Foundation Classes for the graphical user interface (GUI) and OpenGL Optimizer for $3 \mathrm{D}$ visualization.

The model. The virtual models of the anatomical structures are obtained elaborating the images acquired with an accurate preoperative CT/MRI examination of the patient's limb. The examination is done with the patient in a standard position. The total number of slice is around 120 divided in two sets of different acquisition parameters: one group is $1 \mathrm{~mm}$ thickness $1 \mathrm{~mm}$ scan spacing from the upper side of patella to beneath the tibial spine (around 80 slices); the other group is $1 \mathrm{~mm}$ thickness $3 \mathrm{~mm}$ scan spacing in the adjacent areas both of the femur and of the tibia. The examination is safe (dose on critical organs $<0.2 \mu \mathrm{Gy}$ ) and fast (time in helical 
machine $<5 \mathrm{~min}$ ). The images processing consists in a first segmentation phase performed semi-automatically with an algorithm that provides contour tracking by means of gradient-of-gaussian filtering and in a successive 3D computer reconstruction phase using the marching cube algorithm.

CAD drawings of the digitizer and of the cutting guide are also drawn in the scenario.

The registration module. The intraoperative registration module matches the coordinate system of the virtual scenario with the real position of the patient and tools in the operating room. Pre-operatively, an operator identifies, on the virtual model, areas that will be accessible during surgery and intra-operatively the surgeon acquires, with a digitizer, points in the correspondent areas on the bone. Registration is performed by means of an ICP-based non-fiducial algorithm.

The tracking module. The tracking module elaborates the information retrieved by the optical localizer to reproduce in real time the surgical environment in the virtual scenario.

The user interface. The virtual scenario of the operating room is shown on a computer display, which contains four windows: the first is the external viewpoint window, and it displays the surgical scene as viewed from an external viewpoint. In this window the main objects in the operating room are displayed together with the anatomical structures and the surgical tools; the view may be adjusted according to surgeon preference by changing camera position, orientation and magnification.

The remaining three windows show the anatomical components present in the scene and the trajectory of the cutting plane drawn from different point of view and in different graphical style: in the lateral projection window and the frontal projection window, the bones are shown in translucent wire-frame mode while in the Solid $3 D$ view they are shown in solid mode (fig.2).

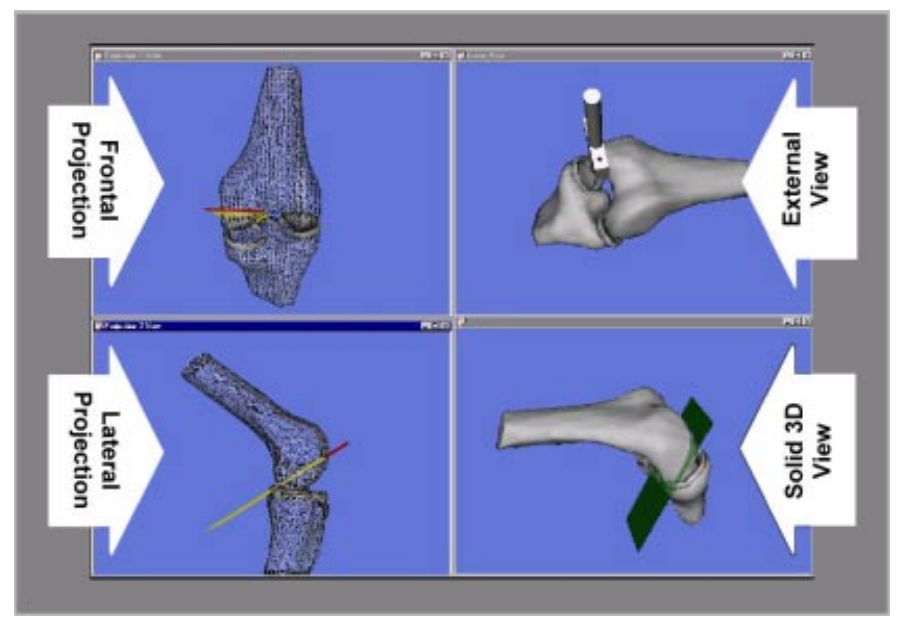

Fig. 2. The user interface 


\section{Description of the Assisted Surgical Procedure}

The surgeon makes a medial parapatellar skin incision of $8-10 \mathrm{~cm}$, extending from the proximal border of the patella to the proximal tibial region.

After the knee exposure, the two sensorized frames are fastened to femur and tibia with two fiches.

Next step is the registration phase: the surgeon acquires, with the optical localizer's digitizer, points in the bone surface, in areas corresponding to the ones preoperatively identified on the virtual model. Using this set of points the system provides to perform the registration.

After the registration phase, the system displays the virtual scenario reproducing in real time the surgical environment: the sensorized cutting guide is shown together with the two bones.

Now the surgeon can track in real-time the placement of the cutting plane on the bone in the enhanced reality scenario, so that he can choose the desired cutting guide position and orientation with respect to the anatomical structures before the cut is effectively done.

The sensorized cutting guide is used by the surgeon to make the horizontal tibial cut and the femur first distal cut.

\section{Discussion and Conclusions}

In this paper we have presented new minimally invasive computer-assisted procedure for UKA relying on an augmented reality navigation system and on a reduced set of easily usable surgical instruments.

The procedure that we have described is an original application of navigation systems to UKA, and thus can hardly be compared with previous literature.

Preliminary tests done to see the accuracy of the navigation system, show that the overall system error, defined as the average difference between coordinates in the virtual scene and the corresponding coordinates in the real world, is about $2 \mathrm{~mm}$, whereas the system update rate, defined as the inverse of the time needed by the system to acquire all data and update the virtual scene accordingly, is $13 \mathrm{~Hz}$.

Early evaluation of the surgical system done using artificial model of the human knee shows that the computer assisted UKA is as time-consuming as the traditional technique: this is probably due to the fact that the registration procedure is completed in less than 10 minutes, and the reduced and simple set of surgical instruments coupled with the enhanced field of view diminish the time required to take the intraoperative decisions.

The new set of instruments can be easily sterilised.

The UKA procedure presented allows the surgeon to reduce the skin incision size from $20 \mathrm{~cm}$ to $8-10 \mathrm{~cm}$; the navigation system solves the problems due to a restricted knee exposure providing the surgeon with the enlarged view of the patient's limb on the 3D virtual model obtained from a CT/MRI accurate study. The first prototype system has been favorably evaluated by a selected group of skilled orthopedic 
surgeons. At this moment we are performing tests to evaluate the final accuracy and repeatability of the surgical decisions.

\section{References}

1. Ryd L, Boegård T, Egund N, Lindstrand A, Selvik G, Thorngren KG: Migration of the tibial component in successful unicompartmental knee arthroplasty. A clinical, radiographic and roentgen stereophotogrammetric study. Acta Orthop. Scand. 54: 408, 1983

2. Ryd L: Micromotion in knee arthroplasty. A Roentgen Stereophotogrammetric Analysis of tibial component fixation. Acta Orthop. Scand. 57 (Suppl. 220): 1, 1986

3. Lindstrand A, Stenström A, Egund N: The PCA unicompartmental knee: a 1-4 year comparison of fixation with or without cement. Acta Orthop. Scand. 59(6): 695, 1988

4. Goodfellow J.W., Kershaw C.J., D'A Benson M.K., O'Connor J.J.: The Oxford knee for unicompartmental osteoarthritis. J Bone Joint Surg [Br] 1988;70-B:692 - 701.

5. Repicci J.A., Eberle R.W.: Minimally Invasive Surgical Technique for Unicondylar Knee Arthroplasty. J South Orthop Assoc 1999;8(1):20-27

6. P. Dario, M.C. Carrozza, M. Marcacci, S. D'Attanasio, B. Magnani, O. Tonet and G. Megali, (2000) “A Novel Mechatronic Tool for Computer-Assisted Arthroscopy”. IEEE Transactions on Information Technology in Biomedicine, 4(1), 15 - 29. 